\title{
Quality Factor Measurements at NTF
}

K. Vaziri, F. Krueger, T. Kroc, G. Lauten, A. Lennox and T. Leveling

Fermi National Accelerator Laboratory

P.O. Box 500, Batavia, Illinois 60510

December 1993 


\section{Disclaimer}

This report was prepared as an account of work sponsored by an agency of the United States Government. Neither the United States Government nor any agency thereof, nor any of their employees, makes any warranty, express or implied, or assumes any legal liability or responsibility for the accuracy, completeness, or usefulness of any information, apparatus, product, or process disclosed, or represents that its use would not infringe privately owned rights. Reference herein to any specific commercial product, process, or service by trade name, trademark, manufacturer, or otherwise, does not necessarily constitute or imply its endorsement, recommendation, or favoring by the United States Government or any agency thereof. The views and opinions of authors expressed herein do not necessarily state or reflect those of the United States Government or any agency thereof. 


\section{Quality Factor Measurements at NTF Kamran Vaziri and Fred Krueger \\ Tom Kroc, Gary Lauten, Arlene Lennox, Tony Leveling \\ December 17, 1993}

\section{Introduction}

The dose equivalent rate in the radiation field outside of the polydoor at the Neutron Therapy Facility 1 (see Figure 1.) has been measured, using a Chipmunk, assuming a quality factor (QF) of 5 , to be 25 $\mathrm{mrem} / \mathrm{hr}$. This kind of dose rate if true introduced occupancy restrictions and NTF is operating under an exemption. Based on the previous CR-39 studies $^{2}$ of the neutron field around NTF, and the amount of shielding around the NTF, it was difficult to believe that a significant neutron field exists in this area, and contributes to the measured dose rate. If the field was mostly due to gamma rays the $\mathrm{QF}$ setting on the Chipmunk could be reliably set to a value of one. One method of obtaining a qualitative understanding of the relative abundance of neutron and gamma contribution to the absorbed doses, is to measure the quality factor for the field. This was determined using a recombination chamber.

The recombination chamber is a gas filled ion chamber that can measure the average quality factor of a radiation field of unknown composition and energy spectrum ${ }^{3,4}$. The response of the recombination chamber can be described ${ }^{5}$ as a function of the bias across it's plates as

$$
I=k V^{n},
$$

where $\mathrm{I}$ is the normalized current collected by the chamber, $\mathrm{k}$ is a constant of proportionality, $\mathrm{V}$ is the applied bias and the exponent $\mathrm{n}$ can be related to the quality factor of the field. To use the recombination chamber in an unknown field, one needs to have measured a calibration curve using radiation fields of known quality factor $6,7,8$.

The individual neutron and gamma components of the radiation field were also determined in these studies by use of an Andersson-Braun counter (SNOOPY ${ }^{9}$ ) to measure the dose equivalent rate due to neutrons, and a CutiePie ${ }^{10}$ ion chamber to measure the gamma dose rate. The neutron dose equivalent rate in this area of NTF has been estimated by Vylet ${ }^{11,2}$, and is consistent with the present measurements.

\section{Calibration of the Recombination Chamber}

A schematic drawing of the recombination chamber setup is shown in Figure 2. The calibration was performed at the neutron irradiation area of the Radiation Physics Calibration Facility (RPCF). The recombination chamber was placed on an aluminum ladder, half way between the ceiling and floor, to minimize the effects of room scattering. Radiation with at least two different known quality factors need to be measured to produce the linear calibration curve. A gamma ray source $\left({ }^{60} \mathrm{Co} ; 60-4.3-2\right)$, and a mixed neutron-gamma source $\left({ }^{241} \mathrm{Am}-\mathrm{Be} ; 241 \mathrm{Be}-7.2-1\right)$ were used to obtain the calibration reference points. The standard quality factor for gamma rays (of any energy) is assumed to be one. The quality factor due to neutrons is a sensitive function of energy ${ }^{12}$.The average quality factor for the mixed ${ }^{241} \mathrm{AmBe}$ source was calculated to be 6.6 using

$$
Q F=\sum_{i=1}^{n} \frac{(\text { Dose })_{i}}{\text { Total Dose }}(Q F)_{i}
$$

where (Dose $)_{i}$, and $(\mathrm{QF})_{\mathrm{i}}$ are dose and the quality factor due to the $\mathrm{i}^{\text {th }}$ component of the field. Only neutron and gamma radiation fields are of interest in this note. The quality factor for the predominant neutron component of the AmBe source was taken to be 7.912. The results of the calibration measurements are given in Tables I and II. The last column in each table shows the response of the recombination chamber to the radiation field which is calculated using

$$
\text { Response }=\frac{I_{\mathcal{V}}}{I_{\text {saturation }}},
$$


where $I_{v}$ is the measured current at a bias setting of $V$, and $I_{\text {saturation }}$ is the measured current at saturation bias which is taken as -1200 volts. The data columns labeled Data\#1, Data\#2, and Data\#3 are one minute charge integrations and the net charge for each interval is

$$
\begin{aligned}
& Q 1(n \text { Coulombs })=\text { Data\# } 2-\text { Data\#1, } \\
& Q 2(n \text { Coulombs })=\text { Data\#3 }- \text { Data\# } 2 .
\end{aligned}
$$

The recombination chamber response for cobalt and $\mathrm{AmBe}$ sources, at different bias settings are plotted in Figures 3 and 4. The power law fit with the fit parameters are also displayed on each plot. Figure 5 shows the exponent $n$ (defined in Equation 1) plotted against the quality factor for the two sources. The linear fit to these two points, as shown on the plot, provides the calibration curve for the recombination chamber. It is in good agreement with previous calibration measurements ${ }^{7}$. The quality factor for any unknown field is obtained by measuring the response curve. From the fit of the response to Equation 1, the exponent $n$ can be obtained, and the quality factor for the unknown field can be read off the calibration curve Figure 5.

\section{Measurements}

The main part of the measurements was done with the recombination chamber positioned at the geometric center of the polydoor at NTF, and the Chipmunk detector located on the floor outside the polydoor. The Chipmunk detector was used to provide a redundant dose normalization factor. As mentioned a SNOOPY detector was used to measure the neutron dose equivalent rate, and a CutiePie detector was used to extract the gamma ray component of the field.

To simulate the worst case operation of NTF, a large $\left(24 \mathrm{X} 24 \mathrm{~cm}^{2}\right)$ collimator was used, and the beam was scattered off a 5 gallon carboy containing water. Each run lasted about 2.3 minutes and delivered 0.65 monitor units.

\section{III.A RF and X-ray Backgrounds}

A large RF background can inundate the recombination chamber and make the measurements useless. The RF levels in the area around NTF have been surveyed in the past ${ }^{13}$, using an RF meter. However, that measurement was only concerned with the health hazards, and the qualitative conclusion was that the levels were below the health hazard threshold. We measured the background using the recombination chamber to see if there were any X-ray and RF pickup effects. This was done a day before the actual measurements and the neutron beam had been off for at least a day. The recombination chamber was operated at the recombination voltage $(-65 \mathrm{~V})$ and the saturation voltage $(-1200 \mathrm{~V})$ and also with and without a Faraday cage surrounding the chamber. Our background measurements revealed three important points: First, there was no measurable RF interference. Second, the measurements showed that the background levels are the same as those measured at RPCF. Third, the background was about $0.2 \%$ of the dose rate we expected to measure with the beam on, and thus of negligible effect.

\section{III.B Background measurements}

During the day of studies, with the neutron beam off, a background measurement was performed with all the detectors used in this study. The results are shown in Table III. Later measurements with the neutron beam showed that the measured backgrounds for the CutiePie, SNOOPY, and the recombination chamber were less than $0.5 \%$ of the dose that was measured with the beam on. After the measurements were completed the background was measured again using the Chipmunk and the recombination chamber. The results are shown in Table IV. The Chipmunk's pre- and postirradiation backgrounds are the same, the recombination chamber measured a higher post-irradiation background due to it's sensitivity to residual induced activity.

\footnotetext{
* The neutron beam is calibrated such that one monitor unit delivers to tissue (or its equivalent) one Gray at a depth of $10 \mathrm{~cm}$ when a $10 \times 10 \mathrm{~cm}^{2}$ collimator is used.
} 


\section{III.C Beam-On Measurements}

During the beam-on measurements the polydoor was surveyed with a CutiePie; the exposure rate was highest in the middle of the door $\left(4-6 \mathrm{mR} / \mathrm{hr}^{* *}\right)$. A dose integration was done with the CutiePie, SNOOPY and a Chipmunk at the middle of the door and on the floor. The results of these measurements are given in Tables V and VI. Data from Radiation Physics Note $86^{14}$, and additional tests ${ }^{15}$ show that the CutiePie has an efficiency of $100 \%$ for the gamma rays, and about $23 \%$ for the detection of neutrons. Actually the neutron detection efficiency of the CutiePie increases with energy and $23 \%$ is a conservative estimate which is true for neutrons of average energy $4.1 \mathrm{MeV}$. The SNOOPY is sensitive to neutrons only, and measures the dose equivalent rate directly ${ }^{9}$. As shown in Table VI, the neutron dose equivalent on the floor is 1.5 times that at the middle of the door, but the absolute value of the neutron dose equivalent is about $20 \%$ of the gamma dose equivalent. The SNOOPY's resolving time is $1 \mu \mathrm{s}$. For the LINAC's $57 \mu$ s pulse width, and frequency of $15 \mathrm{~Hz}$, the dead time correction is $0.2 \%$ for the SNOOPY measurement at the center of the door. The correction for the dose rate on the floor is $\mathbf{0 . 3 5 \%}$. No corrections were applied to the data, since the SNOOPY dead time correction is performed internally.

The recombination chamber's response was measured at 17 different bias setting from -30 volts to -1200 volts, as shown in Table VII. For each one of these bias settings one run was integrated. During these measurements the Chipmunk was placed on the floor and simultaneously integrated the runs, using a scaler. The Chipmunk's measurements indicated the LINAC delivered a fixed dose each run corresponding to the same number of protons. It is seen from the data that the dose delivered was very stable, and the small variation in the chipmunk count-rate ( \pm 2 counts/run) was due to variation in the background and the reaction time in manually starting and stopping the scaler.

\section{Analysis of Data}

A direct way to calculate the quality factor for a radiation field is to measure the dose rate of the individual components of the field, and calculate the composite quality factor using equation 2 , rewritten here as:

$$
Q F=\frac{D_{\gamma}}{D_{\gamma}+D_{n}} Q F_{\gamma}+\frac{D_{n}}{D_{\gamma}+D_{n}} Q F_{n}
$$

where $D_{\gamma}$ and $D_{n}$ are the gamma and neutron absorbed dose rates. $Q F_{\gamma}$ is 1.0 by convention and $Q F_{n}$ is determined by measuring the mean energy of the neutrons outside the polydoor at NTF. Previous measurements ${ }^{11}$ showed the neutron spectrum peaks at $150 \mathrm{keV}$. The quality factor of 150 $\mathrm{keV}$ neutrons is 8.5 as shown ${ }^{4}$ in Figure 6 . The dose rate measured by the CutiePie can be described by

$$
D_{\text {CutiePie }}=D_{\gamma} \times \varepsilon_{\gamma}+D_{n} \times \varepsilon_{n}
$$

from which we can obtain $D_{\gamma}$. The detection efficiencies $\varepsilon_{\gamma}$ and $\varepsilon_{\mathrm{n}}$ are 1.0 and 0.23 , respectively. From Table V we can obtain $D_{\text {CutiePie }}$, which is $0.1579 \mathrm{mrad} /$ run in the middle of the door and 0.1535 $\mathrm{mrad} /$ run on the floor. From Table VI, $D_{n}$ is $0.0033 \mathrm{mrad} / \mathrm{run}$ in the middle of the door and 0.0049 $\mathrm{mrad} /$ run on the floor. Using these values in the equation $5, D_{\gamma}$ is calculated to be $0.1569 \mathrm{mrad} / \mathrm{run}$ in the middle of the polydoor, and $0.1520 \mathrm{mrad} / \mathrm{run}$ on the floor. Substituting these values in equation 4 ., we obtain a quality factor 1.2 for the middle of the door and 1.3 for the floor location.

The results of Table VIII are plotted in Figure 7. A power law fit to the points leads to a value of $\mathrm{n}=0.0123$, as indicated on the figure. From the calibration curve, Figure 5 , this gives a quality factor for the radiation field outside of the polydoor of $Q F=1$, consistent with the field arising almost completely from gamma radiation.

This conclusion is verified by the CutiePie and SNOOPY results. The exposure rate measured by the CutiePie is about $0.18 \mathrm{mR} /$ run (Table V) or $0.158 \mathrm{mrad} / \mathrm{run}$ while the SNOOPY registered 0.042 mrem/run (Table VI) at the same location. If one assumes, based on preliminary measurements of

\footnotetext{
${ }^{* *}$ Exposure rate is expressed in milli-Roentgens $/ \mathrm{hr}$ or $\mathrm{mR} / \mathrm{hr}$.
} 
Vylet, that the average energy of the neutrons is about $150 \mathrm{keV}$, then the QF of the neutron field ${ }^{16}$ is about 8.5 (Figure 6) and the measured neutron absorbed dose is $0.042 / 8.5=0.0049 \mathrm{mrad} / \mathrm{run}$. Thus, the neutrons contribute only about $3 \%$ to the absorbed dose outside of the polydoor. It is entirely reasonable therefore that whenever chipmunks are used to monitor the dose equivalent in this area of NTF, QF be set to 1 .

It might further be pointed out that the results based on the SNOOPY measurements which give neutron dose equivalent of 0.028 to $0.042 \mathrm{mrem} /$ run are in agreement with Vylet's CR-39 film badge results at the same location. A one LINAC run every 2.3 minutes, which is the configuration used during these studies, corresponds to 26 runs/hr. The neutron dose equivalent rates then vary from about 0.7 to about $1 \mathrm{mrem} / \mathrm{hr}$. This is slightly higher but in approximate agreement with the values shown in the table in reference 2.

The quality factor obtained in these measurements, implies - as also seen from the CutiePie and SNOOPY measurements- that the main component of the field outside the NTF polydoor is gamma rays, and a small component is consistent with $150 \mathrm{keV}$ neutrons. Our previous experience with the recombination chamber give a $\pm 5 \%$ statistical error associated with the calibration curve. Typical error associated with the recombination chamber measurements, when operated in the parallel mode, is quoted ${ }^{3}$ to be $25 \%$. In the absence of sufficient statistics and better calibrations and, we adopt this more conservative error, and conclude that the quality factor for the radiation field outside the polydoor of NTF is $1.0 \pm 25 \%$. 


\section{References}

(1) Lionel Cohen and Miguel Awschalom, "The Cancer Therapy Facility at The Ferni National Accelerator Laboratory, Batavia, Illinois: a Preliminary Report," Applied Radiology, November-December, 1976 p51.

(2) Vasek Vylet, "Radiation survey at NTF" , Memo to Arlene Lennox, 9/20/91.

(3) "POLON", Recombination Chamber Type REM-2 Technical Manual, Glinki, 95-958 Bydgoszcz, Poland.

(4) H. W. Patterson and R. H. Thomas, Accelerator Health Physics, Academic Press, New York, 1973.

(5) A. H. Sullivan and J. Baarli, CERN Report No.63-17 (European Organization for Nuclear Research, Geneva, 1963).

(6) F. P. Krueger, Procedures for Measuring Quality Factor Using the Polon Recombination Chamber, November 1993.

(7) J. D. Cossairt, D. W. Grobe, and M. A. Gerardi, TM-1248, March 1984.

(8) C. Moore, Radiation Physics Note 19, June 1977.

(9) F. P. Krueger, Operating Instruction for the ESP/SNOOPY Neutron Survey Meter, June 1992.

(10) Operating instruction for the 2590 series CutiePie Survey Meters, Publication No. 710$715920,8 / 73$ Texas Nuclear Div. G. D. Searle \& Co.

(11) Vasek Vylet, "First guess about the neutron spectrum outside the NTF shielding" Memo to Arlene Lennox, 9/19/91.

(12) ISO/DIS 8529, Neutron reference radiations for calibrating neutron measuring devices used for radiation protection purposes and for determining their response as a function of neutron energy. International Organization for Standardization, 1986, p. 5.

(13) NIOSH, Health hazard Evaluation Report, HETA 87-329-1898. Fermilab, Batavia, Ill. (1988)

(14) F. P. Krueger, Radiation Physics Note 86, June 1990.

(15) Private communication with G. Lauten (AD) and F. Krueger (ES\&H).

(16) National Council on Radiation Protection, Protection against neutron adiation, NCRP Report 38. 


\begin{tabular}{|c|c|c|c|c|c|c|c|}
\hline Bias & Data\#1 & Data\#2 & Data\#3 & Q1(nC) & Q2(nC) & Ave[Q(nC)] & Response \\
\hline-30 & 0.0197 & 0.1547 & 0.297 & 0.135 & 0.1423 & 0.13865 & 0.7013 \\
\hline-40 & 0.0373 & 0.1827 & 0.328 & 0.1454 & 0.1453 & 0.14535 & 0.7352 \\
\hline-65 & 0.0420 & 0.1986 & 0.3541 & 0.1566 & 0.1555 & 0.1561 & 0.7893 \\
\hline-75 & 0.0628 & 0.2209 & 0.3832 & 0.1581 & 0.1623 & 0.1602 & 0.8103 \\
\hline-85 & 0.065 & 0.2285 & 0.392 & 0.1635 & 0.1635 & 0.1635 & 0.827 \\
\hline-100 & 0.0683 & 0.234 & 0.3934 & 0.1657 & 0.1594 & 0.16255 & 0.8222 \\
\hline-200 & 0.094 & 0.2687 & 0.4436 & 0.1747 & 0.1749 & 0.1748 & 0.8842 \\
\hline-300 & 0.0692 & 0.25 & 0.4288 & 0.1808 & 0.1788 & 0.1798 & 0.9095 \\
\hline-400 & 0.0682 & 0.2481 & 0.4339 & 0.1799 & 0.1858 & 0.18285 & 0.9249 \\
\hline-500 & 0.073 & 0.2598 & 0.4444 & 0.1868 & 0.1846 & 0.1857 & 0.9393 \\
\hline-600 & 0.0746 & 0.2653 & 0.4565 & 0.1907 & 0.1912 & 0.19095 & 0.9659 \\
\hline-700 & 0.071 & 0.2653 & 0.4587 & 0.1943 & 0.1934 & 0.19385 & 0.9805 \\
\hline-800 & 0.0744 & 0.2706 & 0.4627 & 0.1962 & 0.1921 & 0.19415 & 0.982 \\
\hline 900 & 0.0965 & 0.2888 & 0.4874 & 0.1923 & 0.1986 & 0.19545 & 0.9886 \\
\hline-1000 & 0.0829 & 0.2832 & 0.4823 & 0.2003 & 0.1991 & 0.1997 & 1.0101 \\
\hline-1100 & 0.074 & 0.2718 & 0.4682 & 0.1978 & 0.1964 & 0.1971 & 0.997 \\
\hline-1200 & 0.1326 & 0.3299 & 0.528 & 0.1973 & 0.1981 & 0.1977 & 1 \\
\hline
\end{tabular}

Table I. Recombination chamber calibration data with the AmBe mixed neutron and gamma source. The background for this run was measured to be $0.00087 \mathrm{nC} / \mathrm{min}$.

\begin{tabular}{|c|c|c|c|c|c|c|c|}
\hline Bias & Data \#1 & Data \#2 & Data \#3 & Q1(nC) & Q2(nC) & Ave [Q(nC)] & Response \\
\hline-30 & 0.248 & 1.284 & 2.308 & 1.036 & 1.024 & 1.03 & 0.9445 \\
\hline-40 & 0.257 & 1.314 & 2.36 & 1.057 & 1.046 & 1.0515 & 0.9642 \\
\hline-65 & 0.247 & 1.304 & 2.36 & 1.057 & 1.056 & 1.0565 & 0.9688 \\
\hline-75 & 0.315 & 1.373 & 2.433 & 1.058 & 1.06 & 1.059 & 0.9711 \\
\hline-85 & 0.271 & 1.34 & 2.393 & 1.069 & 1.053 & 1.061 & 0.9729 \\
\hline-100 & 1.743 & 2.818 & 3.878 & 1.075 & 1.06 & 1.0675 & 0.9789 \\
\hline-200 & 0.362 & 1.438 & 2.518 & 1.076 & 1.08 & 1.078 & 0.9885 \\
\hline-300 & 0.71 & 1.797 & 2.872 & 1.087 & 1.075 & 1.081 & 0.9913 \\
\hline-400 & 0.826 & 1.9 & 2.992 & 1.074 & 1.092 & 1.083 & 0.9931 \\
\hline-500 & 0.358 & 1.446 & 2.537 & 1.088 & 1.091 & 1.0895 & 0.9991 \\
\hline-600 & 1.182 & 2.261 & 3.355 & 1.079 & 1.094 & 1.0865 & 0.9963 \\
\hline-700 & 2.138 & 3.224 & 4.314 & 1.086 & 1.09 & 1.088 & 0.9977 \\
\hline-800 & 0.48 & 1.572 & 2.661 & 1.092 & 1.089 & 1.0905 & 1.0000 \\
\hline 900 & 1.519 & 2.611 & 3.701 & 1.092 & 1.09 & 1.091 & 1.0005 \\
\hline-1000 & 2.415 & 3.497 & 4.59 & 1.082 & 1.093 & 1.0875 & 0.9972 \\
\hline-1100 & 0.644 & 1.743 & 2.824 & 1.099 & 1.081 & 1.09 & 0.9995 \\
\hline-1200 & 0.444 & 1.535 & 2.625 & 1.091 & 1.09 & 1.0905 & 1.0000 \\
\hline
\end{tabular}

Table II. Recombination chamber calibration data with the ${ }^{60} \mathrm{Co}$ source. The background for this run was measured to be $0.00087 \mathrm{nC} / \mathrm{min}$. 


\begin{tabular}{|c|c|c|c|c|}
\hline Instrument & Location & Time(min) & Reading & Units \\
\hline Cutie Pie & Center of Door & 10. & 0.001 & $\mathrm{mR}$ \\
\hline Chipmunk & Center of Door & 10. & 49 & counts \\
\hline SNOOPY & Floor & 10. & $2.34 \mathrm{E}-4$ & mrem \\
\hline Chipmunk & floor & 10. & 48 & counts \\
\hline Rec. Cham. (-65V) & Center of Door & 10. & $-33 \mathrm{E}-4$ & nCoul. \\
\hline Rec. Cham. $(-1200 \mathrm{~V})$ & Center of Door & 10. & $-39 \mathrm{E}-4$ & nCoul. \\
\hline SNOOPY & Center of Door & 10. & $1.17 \mathrm{E}-4$ & mrem \\
\hline
\end{tabular}

Table III. Background measurements outside the polydoor.

\begin{tabular}{|l|l|c|c|c|}
\hline \multicolumn{1}{|c|}{ Instrument } & Location & Time(min) & Reading & Units \\
\hline Chipmunk & Floor & 10. & 46 & counts \\
Rec. Cham. (-65V) & Center of Door & 10. & -0.0155 & nCoul. \\
Chipmunk & Floor & 10. & 47 & counts \\
Rec. Cham. (-1200V) & Center of Door & 10. & -0.0156 & nCoul. \\
\hline
\end{tabular}

Table IV. Post irradiation background measurements outside the polydoor at NTF.

\begin{tabular}{|l|c|}
\hline Location & Dose(mR/run) \\
\hline Center of Door & 0.18 \\
\hline Floor & 0.175 \\
\hline
\end{tabular}

Table V. CutiePie dose integration outside the polydoor.

\begin{tabular}{|c|c|c|c|c|}
\hline $\begin{array}{c}\text { Monitor } \\
\text { (Dose) }\end{array}$ & $\begin{array}{c}\text { Chipmunk } \\
\text { Location }\end{array}$ & $\begin{array}{c}\text { Counts } \\
\text { (gross) }\end{array}$ & $\begin{array}{c}\text { SNOOPY } \\
\text { Location }\end{array}$ & $\begin{array}{c}\text { Dose } \\
\text { (mrem) }\end{array}$ \\
\hline $0.62 \mathrm{~Gy}$ & $\begin{array}{c}\text { Center of } \\
\text { Door }\end{array}$ & 422 & Floor & $4.24 \mathrm{E}-2$ \\
\hline $0.62 \mathrm{~Gy}$ & Floor & 274 & $\begin{array}{c}\text { Center of } \\
\text { Door }\end{array}$ & $2.81 \mathrm{E}-2$ \\
\hline
\end{tabular}

Table VI: Simultaneous dose measurements with Chipmunk and SNOOPY. Each measurement is a one-run integration. 


\begin{tabular}{|c|c|c|c|}
\hline Recombination & \multicolumn{2}{|c|}{ Chamber } & Chip munk \\
\hline (Volts) & (nCoul.) & 274 & Time(sec) \\
\hline-30 & 0.5360 & 274 & 223 \\
\hline-40 & 0.5422 & 272 & 248 \\
\hline-65 & 0.5463 & 271 & 217 \\
\hline-75 & 0.5481 & 270 & 217 \\
\hline-85 & 0.5503 & 274 & 210 \\
\hline-100 & 0.5526 & 274 & 251 \\
\hline-200 & 0.5591 & 276 & 250 \\
\hline-300 & 0.5603 & 276 & 243 \\
\hline-400 & 0.5610 & 275 & 248 \\
\hline-500 & 0.5606 & 274 & 258 \\
\hline-600 & 0.5622 & 275 & 252 \\
\hline-700 & 0.5657 & 275 & 250 \\
\hline-800 & 0.5654 & 276 & 251 \\
\hline-900 & 0.5640 & 273 & 252 \\
\hline-1000 & 0.5646 & 276 & 252 \\
\hline-1100 & 0.5651 & 274 & 253 \\
\hline-1200 & 0.5641 &.-- & 254 \\
\hline-800 & 0.5622 & 276 & -- \\
\hline-85 & 0.5499 & 274 & 252 \\
\hline-65 & 0.5495 & & 250 \\
\hline
\end{tabular}

Table VII: Simultaneous dose measurements with recombination chamber and Chipmunk. Each measurement is a one-run integration.

\begin{tabular}{|c|c|c|c|c|}
\hline Blas & Data \#1 & Data \#2 & Q1(nC) & Response \\
\hline-30 & 0.0011 & 0.5371 & 0.536 & 0.9502 \\
\hline-40 & 0.0003 & 0.5425 & 0.5422 & 0.9612 \\
\hline-65 & 0.0002 & 0.5465 & 0.5463 & 0.9684 \\
\hline-75 & 0.0019 & 0.5506 & 0.5487 & 0.9727 \\
\hline-85 & 0.0012 & 0.5515 & 0.5503 & 0.9755 \\
\hline-100 & 0.0018 & 0.5544 & 0.5526 & 0.9796 \\
\hline-200 & 0.0025 & 0.5616 & 0.5591 & 0.9911 \\
\hline-300 & 0.0018 & 0.5621 & 0.5603 & 0.9933 \\
\hline-400 & 0.0006 & 0.5616 & 0.561 & 0.9945 \\
\hline-500 & 0.0064 & 0.567 & 0.5606 & 0.9938 \\
\hline-600 & 0.003 & 0.5652 & 0.5622 & 0.9966 \\
\hline-700 & 0.0036 & 0.5693 & 0.5657 & 1.0028 \\
\hline-800 & 0.0011 & 0.5665 & 0.5654 & 1.0023 \\
\hline-900 & 0.004 & 0.568 & 0.564 & 0.9998 \\
\hline-1000 & 0.0027 & 0.5673 & 0.5646 & 1.0009 \\
\hline-1100 & 0.0035 & 0.5686 & 0.5651 & 1.0018 \\
\hline-1200 & 0.0013 & 0.5654 & 0.5641 & 1.0000 \\
\hline-800 & 0.0039 & 0.5661 & 0.5622 & 0.9966 \\
\hline-85 & 0.0026 & 0.5525 & 0.5499 & 0.9748 \\
\hline-65 & 0.0038 & 0.5533 & 0.5495 & 0.9741 \\
\hline
\end{tabular}

Table VIII. Recombination chamber data and response from the NTF measurements. 


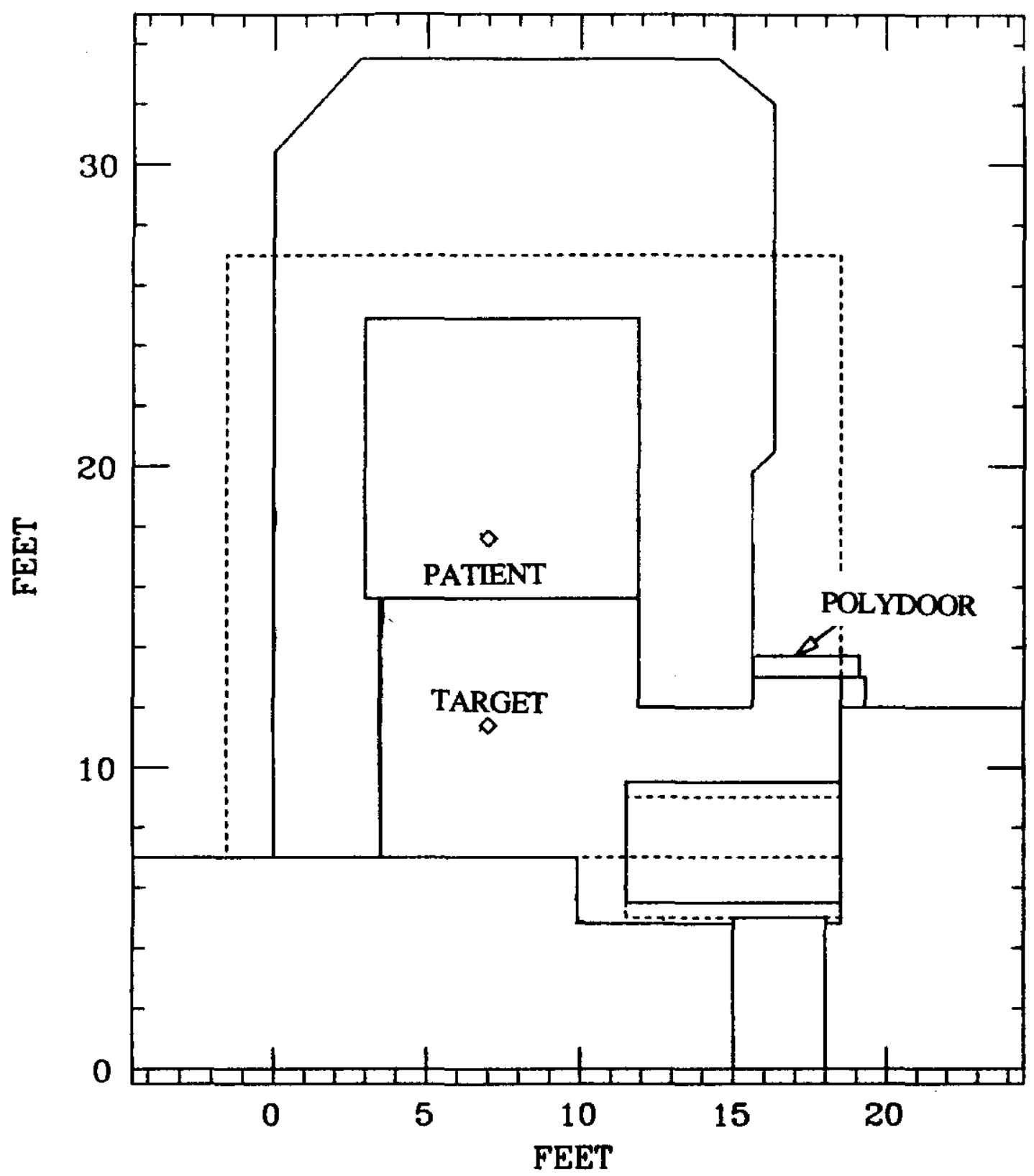

Figure 1. Layout of the NTF. The neutron production target and the treatment room where the phantom was located are below the ground floor and the polydoor was located on the ground floor. 


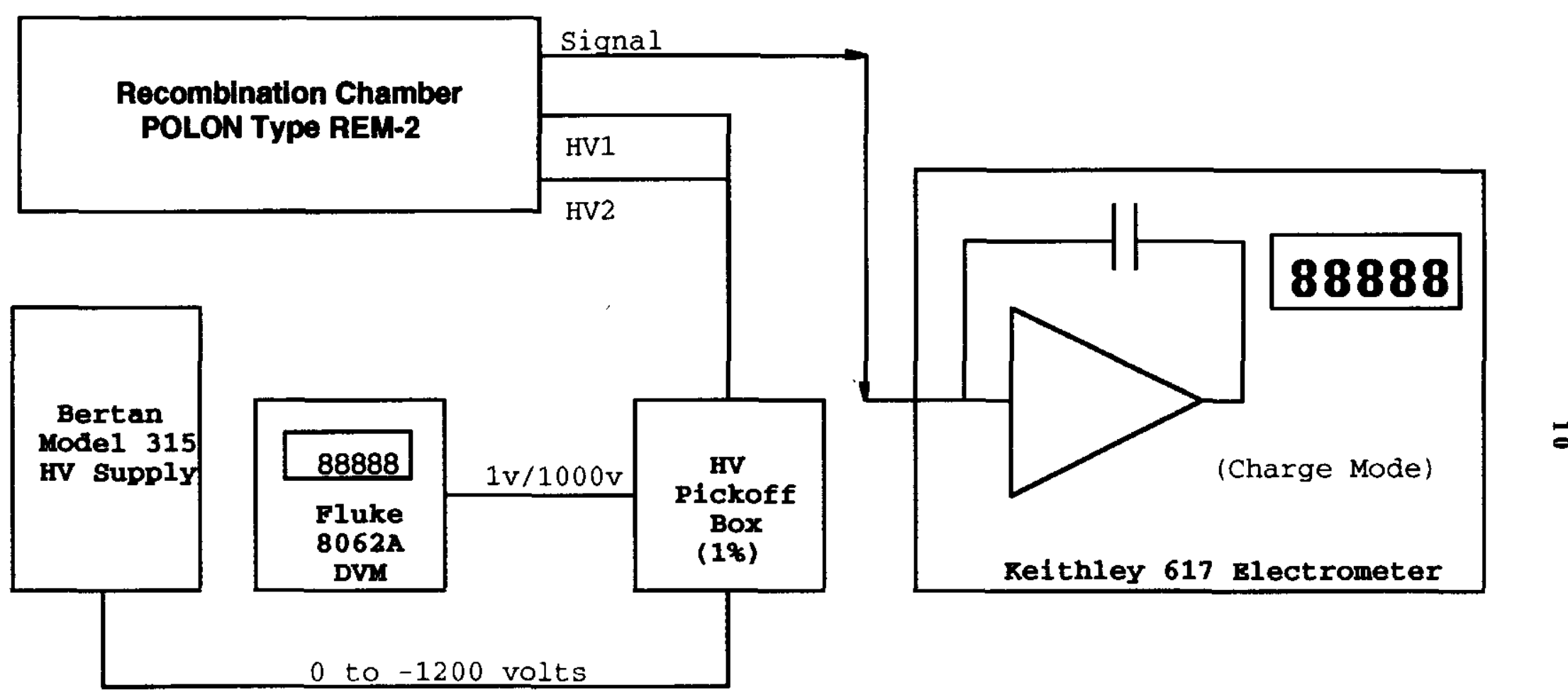

Figure 2. Schematic diagram of the recombination chamber setup for quality factor measurements. 


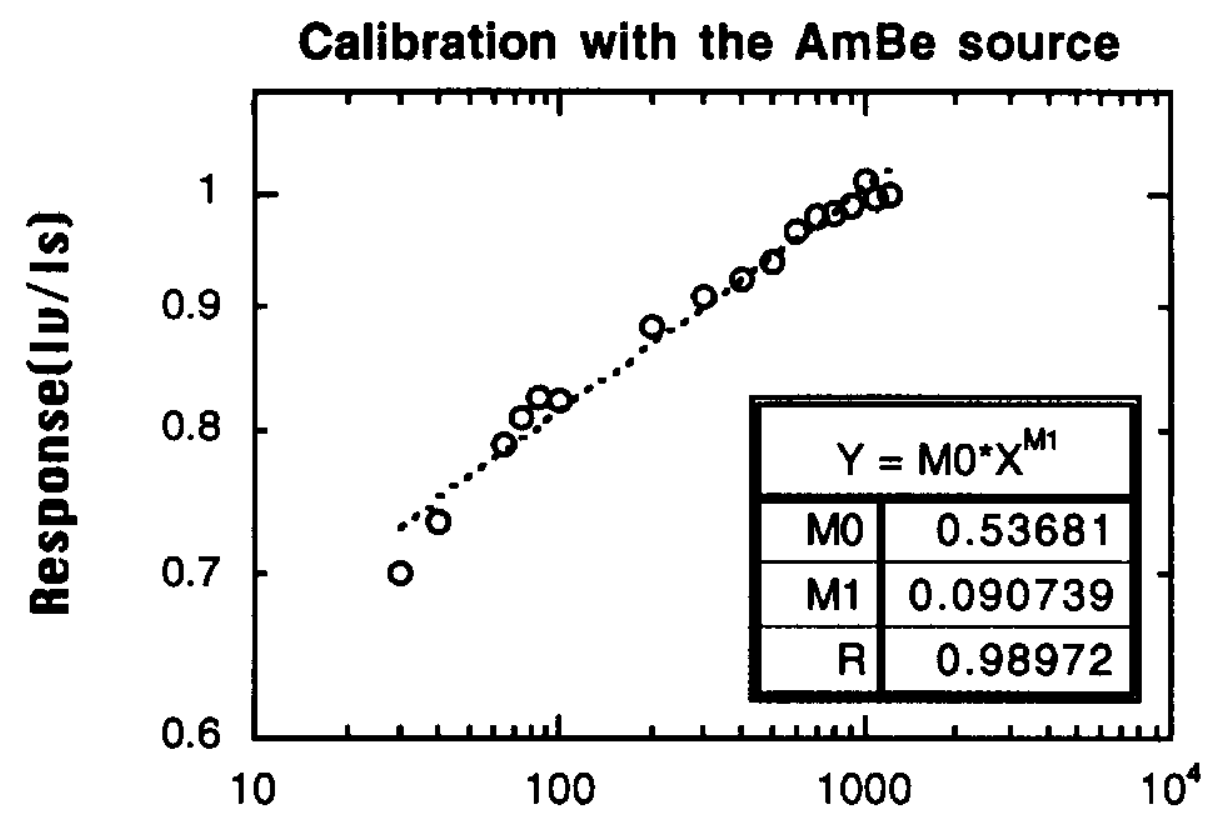

Figure 3. Response of the recombination chamber as a function of its bias settings to the AmBe source.

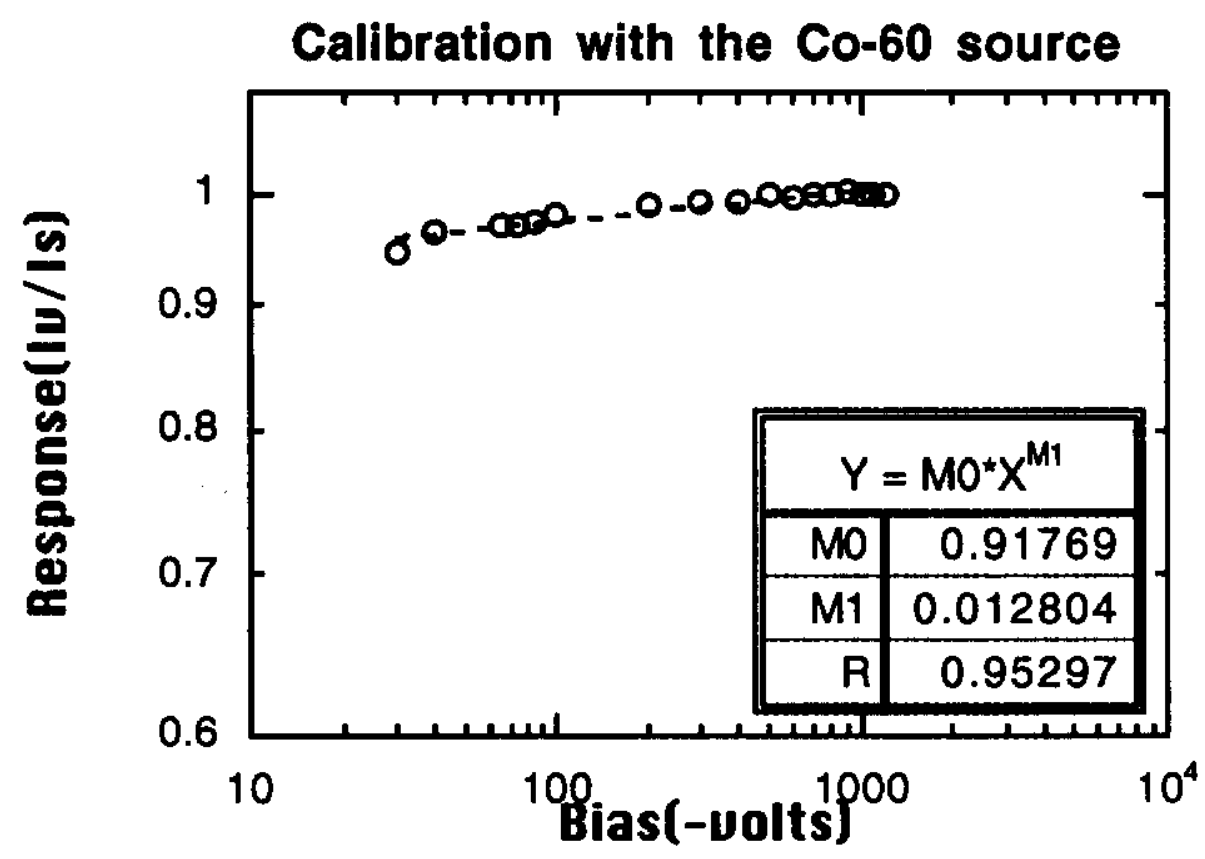

Figure 4. Response of the recombination chamber as a function of its bias settings to the ${ }^{60} \mathrm{Co}$ source. 


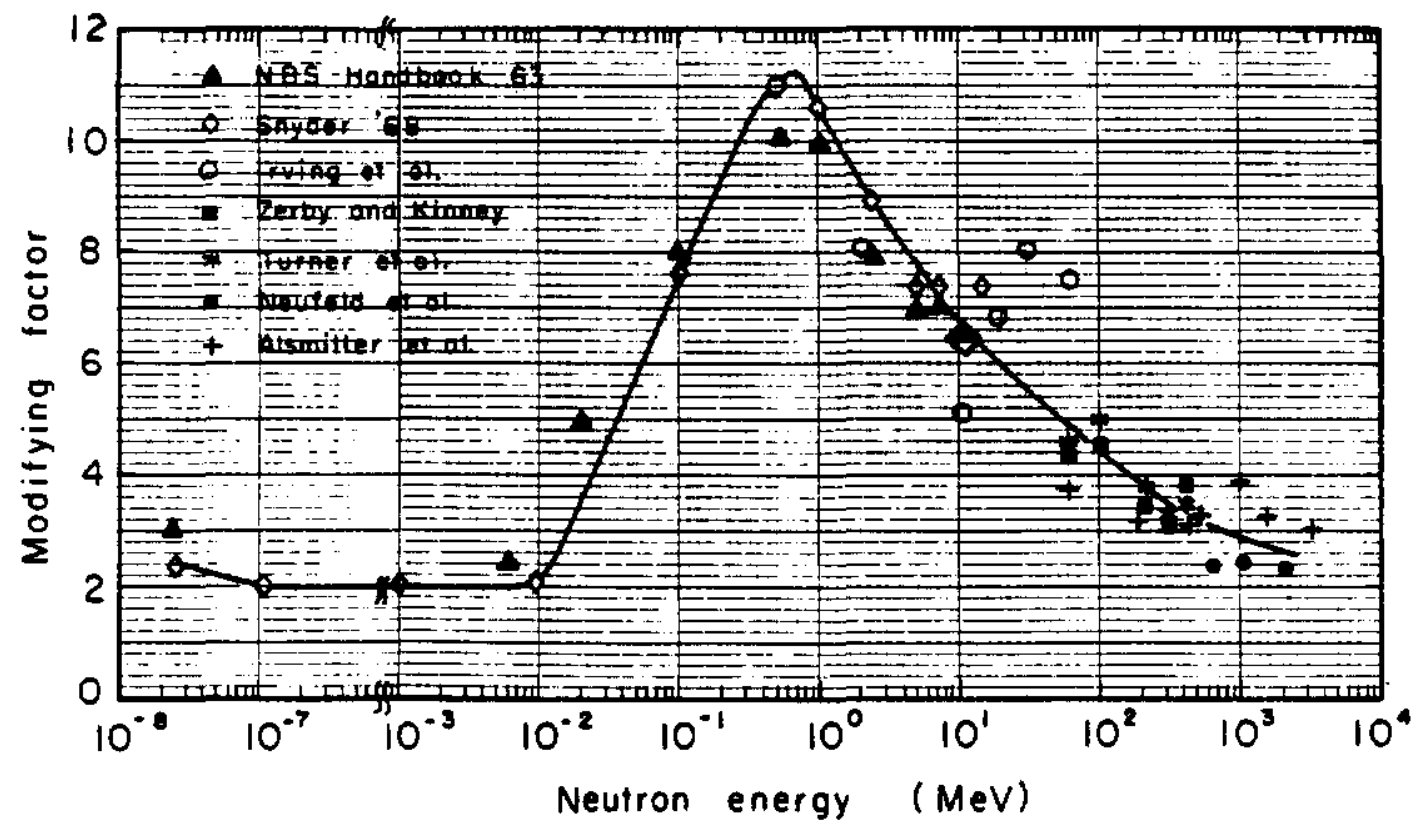

Figure 6. Neutron quality factor as a function of neutron energy.

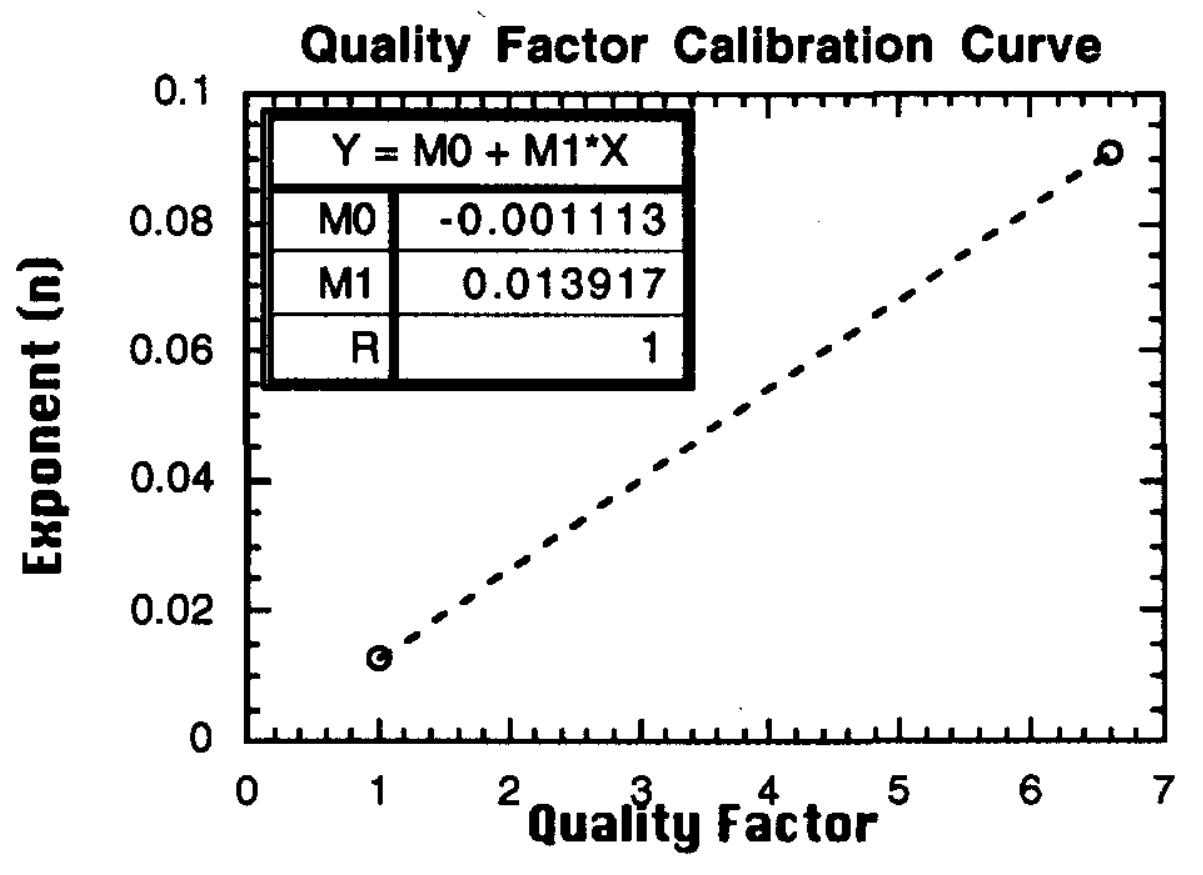

Figure 5. Calibration curve for the recombination chamber. 


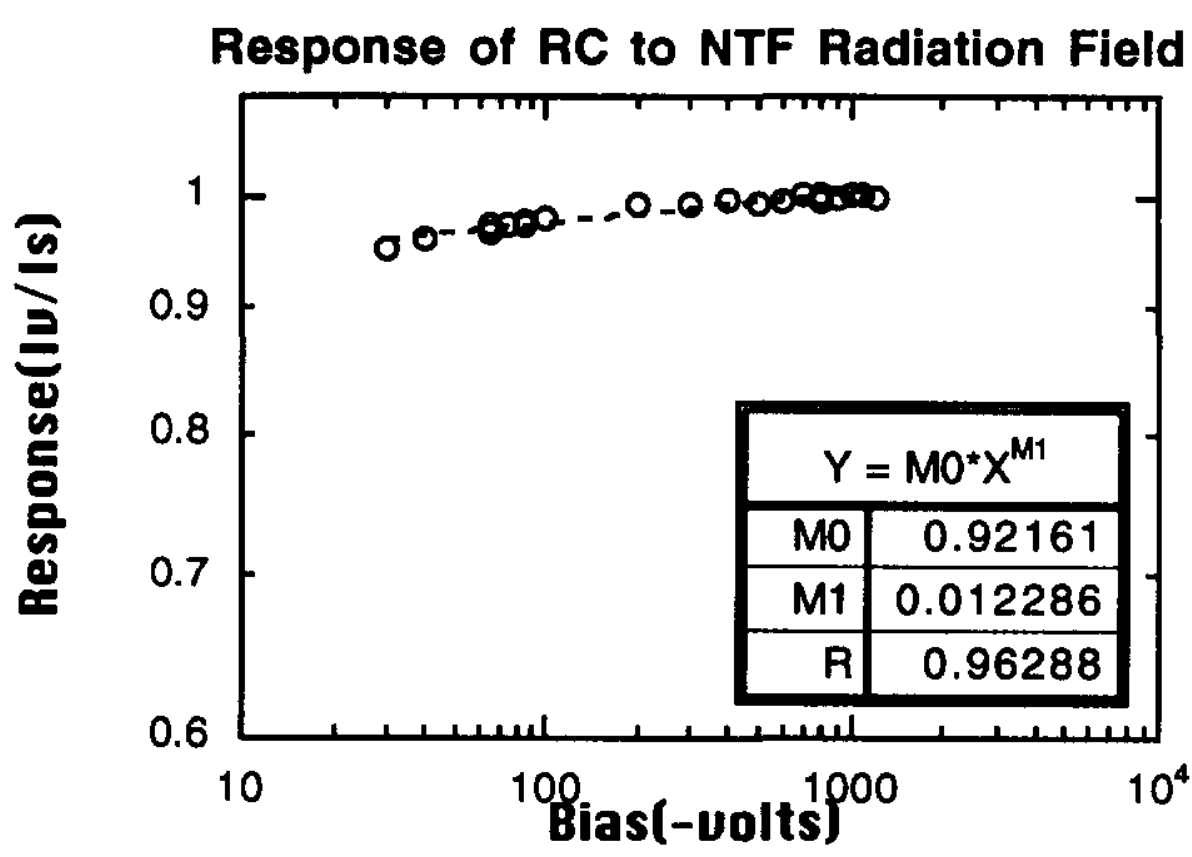

Figure 7. Response of the recombination chamber as a function of its bias settings in the NTF radiation field. 